\title{
Global orthogonality implies local almost-orthogonality
}

\section{J. Michael Wilson}

Abstract. We introduce a new stopping-time argument, adapted to handle linear sums of noncompactly-supported functions that satisfy fairly weak decay, smoothness, and cancellation conditions. We use the argument to obtain a new Littlewood-Paley-type result for such sums.

\section{Introduction.}

First, an apology. The title, though correct, is somewhat misleading. It should be "Global almost-orthogonality implies local almostorthogonality." The present title was chosen for the sake of euphony.

In this paper we present a new Littlewood-Paley type result for linear sums of almost-orthogonal functions. The functions we consider have some decay at infinity and some smoothness. However, neither of these useful properties is assumed to be present in generous amounts. The decay we assume is, in typical cases, no more than will ensure that our functions belong to $L^{1}$, and we do not assume that their gradients decay at any faster rate.

Because of our minimal-decay hypothesis, we are not able to exploit a lemma of Uchiyama [U] which would, in a certain sense, reduce our problem to one in which our functions had compact support. This constraint has required the construction of a new stopping-time argument, one specially adapted to sums of non-compactly-supported functions. We believe that this stopping-time argument is the most significant 
achievement of the present paper.

We shall now be more specific. Let $\mathcal{D}$ denote the usual family of dyadic cubes $I \subset \mathbb{R}^{d}$. We recall that $\mathcal{D}$ has the property that, for any $I$ and $J$ in $\mathcal{D}$, either $I \subset J, J \subset I$, or $I \cap J=\varnothing$. For $I \in \mathcal{D}$, we let $\ell(I)$ denote $I$ 's sidelength and we use $x_{I}$ to mean its center. If $E \subset \mathbb{R}^{d}$ is a measurable set, we let $|E|$ denote $E$ 's Lebesgue measure.

We suppose we are given a family of functions $\left\{\phi_{(I)}\right\}_{I}$, indexed over $I \in \mathcal{D}$. Each $\phi_{(I)} \in\left\{\phi_{(I)}\right\}_{I}$ is smooth and also satisfies

$$
\left|\phi_{(I)}(x)\right|+\ell(I)\left|\nabla \phi_{(I)}(x)\right| \leq|I|^{-1 / 2}\left(1+\frac{\left|x-x_{I}\right|}{\ell(I)}\right)^{-M}
$$

for all $x \in \mathbb{R}^{d}$, where $M$ is a fixed positive number. We furthermore assume that $\left\{\phi_{(I)}\right\}_{I}$ is "almost-orthogonal" in the following precise sense: For every finite linear combination from $\left\{\phi_{(I)}\right\}_{I}$,

$$
f(x)=\sum_{I} \gamma_{I} \phi_{(I)}(x)
$$

the inequality

$$
\int|f|^{2} d x \leq \sum_{I}\left|\gamma_{I}\right|^{2}
$$

holds.

Families satisfying (0.1) and (0.2) are endemic in harmonic analysis. Here is a fast way to get such a family on the line. Let $\psi(x)$ be equal to $\sin (2 \pi x)$ for $x \in[0,1]$ and 0 elsewhere. For $I \in \mathcal{D}$ (on $\mathbb{R}$, mind), set

$$
\psi_{(I)}(x)=|I|^{-1 / 2} \psi\left(\frac{x-x_{I}}{\ell(I)}\right) .
$$

Let $H$ be the Hilbert transform. Then, modulo positive multiplicative constants, $\left\{H\left(\psi_{(I)}\right)\right\}_{I}$ satisfies (0.1) (for $M=2$ ) and (0.2). Inequality (0.1) follows from easy estimates on the Hilbert kernel. Inequality (0.2) calls for some discussion.

An easy way to see that $(0.2)$ holds for $\left\{H\left(\psi_{(I)}\right)\right\}_{I}$ is to use the $L^{2} \longmapsto L^{2}$ boundedness of the Hilbert transform. It is well-known (see [St2, p. 167], or [U, Lemma 3.3]) that $\left\{\psi_{(I)}\right\}_{I}$ satisfies (0.2), modulo a 
multiplicative constant. Thus, for any finite linear sum $\sum_{I} \gamma_{I} H\left(\phi_{(I)}\right)$,

$$
\begin{aligned}
\int\left|\sum_{I} \gamma_{I} H\left(\phi_{(I)}\right)\right|^{2} d x & =\int\left|H\left(\sum_{I} \gamma_{I} \phi_{(I)}\right)\right|^{2} d x \\
& =\int\left|\sum_{I} \gamma_{I} \phi_{(I)}\right|^{2} d x \\
& \leq C \sum_{I}\left|\gamma_{I}\right|^{2} .
\end{aligned}
$$

There is another way to get $(0.2)$, in this particular case. The industrious reader will have noticed that the collection $\left\{H\left(\psi_{(I)}\right)\right\}_{I}$ actually satisfies a stronger condition than (0.1). Indeed

$$
\ell(I)\left|\nabla H\left(\psi_{(I)}\right)(x)\right| \leq A|I|^{-1 / 2}\left(1+\frac{\left|x-x_{I}\right|}{\ell(I)}\right)^{-M-1} .
$$

Furthermore, each $H\left(\psi_{(I)}\right)$ has "cancellation"

$$
\int H\left(\psi_{(I)}\right) d x=0 .
$$

It follows from two lemmas of Uchiyama [U] that any family satisfying (0.1) and (0.3) for some $M>d$, and which also has (0.4), automatically satifies (0.2), modulo a constant. A quick proof of this result depends on a decomposition (due to Uchiyama) that allows one to essentially reduce the problem to one in which the $\phi_{(I)}$ 's have compact supports; for the sake of completeness, we present this argument in an appendix. However, the extra decay in the derivative $(0.3)$ is not necessary for almost-orthogonality: (0.1) and (0.4) suffice ([FJW, p. 11]). Now, the process described above is how families like $\left\{\phi_{(I)}\right\}_{I}$ typically arise: roughly speaking, if one applies a reasonably regular integral operator to a linear sum of wavelet-like functions $\sum_{I} \lambda_{I} \psi_{(I)}$, one ends up with a linear sum from a collection like $\left\{\phi_{(I)}\right\}_{I}$. One then commonly has the problem of relating the size of the new function (in a weighted space, in $L^{p}$, etc.) to the original coefficients $\lambda_{I}$. If the operator is nice enough, then $\left\{\phi_{(I)}\right\}_{I}$ will have (0.3) and (0.4), and one can apply Uchiyama's decomposition to get, in some cases, more general results; such a program is worked out in [W1]. However, if the operator is not quite regular enough, these properties may be destroyed. Just a little bit of oscillation in the operator's kernel function can kill (0.3) (think 
of Bochner-Riesz kernels), and we might lose (0.4) if the operator is not translation-invariant. In such a case, one needs a different approach to handle arbitrary linear sums; and that is the burden of our paper.

We need one more definition before we can state our main result. If $f=\sum \lambda_{I} \phi_{(I)}$ is a finite linear combination from a family $\left\{\phi_{(I)}\right\}_{I}$, and $\varepsilon>0$, we set

$$
g^{*}(f)(x)=\left(\sum_{I} \frac{\left|\lambda_{I}\right|^{2}}{|I|}\left(1+\frac{\left|x-x_{I}\right|}{\ell(I)}\right)^{-(2 M-(d+\varepsilon))}\right)^{1 / 2} .
$$

This is the Littlewood-Paley-type object we will use to bound linear sums from $\left\{\phi_{(I)}\right\}_{I}$. The reader will notice that it is nothing but a realvariable analogue of the familiar $g_{\lambda}^{*}$-function from classical LittlewoodPaley theory (see [St1, Chapter 4]).

Finally, let us recall that a non-negative $\sigma \in L_{\text {loc }}^{1}$ is said to be an $A_{\infty}$ weight (written: $\sigma \in A_{\infty}$ ) if there are positive constants $a$ and $b$ such that for all cubes $Q \subset \mathbb{R}^{d}$ and measurable sets $E \subset Q$,

$$
\frac{\int_{E} \sigma}{\int_{Q} \sigma} \leq a\left(\frac{|E|}{|Q|}\right)^{b} .
$$

Here is our main theorem.

Theorem A. We suppose that $\left\{\phi_{(I)}\right\}_{I}$ satisfies (0.1) and (0.2), for some fixed $M>d / 2$. We also suppose that $\sigma \in A_{\infty}$. Let $0<\varepsilon<$ $2 M-d$ and $0<p<\infty$. There is a constant $C=C(M, p, d, \varepsilon, \sigma)$ such that for every $f=\sum_{I} \lambda_{I} \phi_{(I)}$, a finite linear sum from $\left\{\phi_{(I)}\right\}_{I}$,

$$
\int_{\mathbb{R}^{d}}|f(x)|^{p} \sigma d x \leq C \int_{\mathbb{R}^{d}}\left(g^{*}(f)(x)\right)^{p} \sigma d x .
$$

Our hypothesis that $\left\{\phi_{(I)}\right\}_{I}$ satisfy (0.2) might seem a rather severe requirement. We insist that it is not. First, as noted above, a slight strengthening of the decay and smoothness conditions on $\left\{\phi_{(I)}\right\}_{I}$, when combined with (0.4), yields (0.2) for free, and families meeting these extra conditions pop up fairly often. Second, Theorem A has the happy property of not caring where $\left\{\phi_{(I)}\right\}_{I}$ 's almost-orthogonality comes from: cancellation, Fourier transform tricks, special-functions arcana, etc. This makes Theorem A applicable to the study of operators that are less regular than, say, the Hilbert transform. 
The proof of Theorem A comes by means of a so-called "good$\lambda$ inequality," which in turn depends on the stopping-time argument mentioned above. A few words about this argument are probably in order here. The method of good- $\lambda$ inequalities requires that we be able to analyze the behavior of $\sum_{I} \lambda_{I} \phi_{(I)}$ on arbitrary cubes $J$. This entails splitting $\sum_{I} \lambda_{I} \phi_{(I)}$ into two sums. The first of these, which we will call $\sum_{1}$ for the present, reflects the "coarse structure" of $\sum_{I} \lambda_{I} \phi_{(I)}$ on $J$, and is supposed to be almost constant on $J$. The second sum, $\sum_{2}$, has the information about $\sum_{I} \lambda_{I} \phi_{(I)}$ 's fine structure. The hard work in proving the good $\lambda$ inequality comes in controlling the size of $\sum_{2}$. Now, this control is obtained by applying a stopping-time argument to $\sum_{2}$, which means splitting $\sum_{2}$ itself into two new sums, $\sum_{1}^{*}$ and $\sum_{2}^{*}$. One of these new sums gets handled by means of a "global" result (for us, that will be (0.2)). The other gets treated some other way; in many stopping-time arguments, such as those for dyadic martingales, the second sum disappears. Now, here's our problem. The stoppingtime argument works by analyzing the local behavior of $\sum_{I} \lambda_{I} \phi_{(I)}$; but the functions in $\left\{\phi_{(I)}\right\}_{I}$ have global reach. In order to get a good local estimate, we have to somehow "cut off" the functions $\phi_{(I)}$, but if we do not do this cutting-off correctly, we will lose the property $(0.2)$, which gives us our only hope of controlling $\sum_{2}$.

Our stopping-time argument turns on two main ideas. The first is an appropriate discretization of $\sum_{I} \lambda_{I} \phi_{(I)}$, which is given in Definition 2 below. The second is a special splitting of $\sum_{2}$ into $\sum_{1}^{*}$ and $\sum_{2}^{*}$. The splitting occurs in the proof of the Main Lemma. Neither the splitting nor the discretization seems to have much point without the other; however, in order for the argument to work, these two parts have to fit together as tightly as two Lego blocks. Indeed, our problem actually has three interlocking pieces: one needs to have the right discretization in order to define the right stopping time, in order to apply the right splitting. We believe that that was why this theorem was so hard to prove. We also believe that keeping an image of two (or three) joined Lego blocks in mind will help the reader understand the proof faster.

The organization of the paper is as follows. In Section 1, we give (or repeat) our basic definitions and conventions. In Section 2, we state and prove some technical lemmas. In Section 3 , we state and prove our Main Lemma, from which we obtain the good- $\lambda$ inequality as a corollary. The proof of Theorem A then follows immediately. 


\section{Preliminary definitions and conventions.}

All cubes $I \subset \mathbb{R}^{d}$ are assumed to be dyadic. We denote $I$ 's sidelength by $\ell(I)$. We use $d(x, E)$ to mean the distance between a point $x$ and a set $E$.

We assume that $\left\{\phi_{(I)}\right\}_{I}$, henceforth fixed, is a family of functions satisfying (0.1) and (0.2). We will consider only finite linear sums $\sum_{I} \lambda_{I} \phi_{(I)}$ from $\left\{\phi_{(I)}\right\}_{I}$.

We will make frequent use of two simple facts:

a) if $a$ and $b$ are non-negative numbers and $a \leq C b$, then $(1+a) \leq$ $\max \{1, C\}(1+b) \equiv C^{\prime}(1+b)$;

b) if $I \subset \mathbb{R}^{d}$ is a cube and $\rho>d$ then

$$
\sum_{I^{\prime}: I^{\prime} \subset I}\left(\frac{\ell\left(I^{\prime}\right)}{\ell(I)}\right)^{\rho} \leq C(\rho, d) .
$$

Definition 1. Let $I \subset \mathbb{R}^{d}$ be a cube. $x_{I}$ is I's center. $S(I)$ is the collection of all cubes $I^{\prime}$ such that $I^{\prime} \not \subset I$ (these are the cubes which "surround" $I) . N(I)$ is the collection of cubes $I^{\prime}$ such that $I^{\prime} \subset I$ and $\ell\left(I^{\prime}\right)=0.5 \ell(I)$ (these are the "next generation" of cubes "below" $I$ ).

The first MAIN IDEA is the definition of $F(I)$ : this is how we "discretize" the sum $\sum_{I} \lambda_{I} \phi_{(I)}$.

Definition 2. If $I$ is a cube and $x \in I$, we set

$$
\begin{gathered}
F(I, x)=\sum_{I^{\prime} \in S(I)} \lambda_{I^{\prime}} \phi_{\left(I^{\prime}\right)}(x), \\
G(I, x)=\left(\sum_{I^{\prime} \in S(I)} \frac{\left|\lambda_{I^{\prime}}\right|^{2}}{\left|I^{\prime}\right|}\left(1+\frac{\left|x_{I^{\prime}}-x\right|}{\ell\left(I^{\prime}\right)}\right)^{-(2 M-(d+\varepsilon))}\right)^{1 / 2},
\end{gathered}
$$

where $\varepsilon>0$ is fixed; we do not define $F(I, x)$ or $G(I, x)$ for $x \notin I$. We set $F(I)=F\left(I, x_{I}\right)$ and $G(I)=G\left(I, x_{I}\right)$. 


\section{Definition 3.}

$$
\begin{gathered}
F^{*}(x)=\sup _{I \ni x}|F(I)|, \\
G^{*}(x)=\sup _{I \ni x} G(I), \\
g^{*}(x)=\left(\sum_{I} \frac{\left|\lambda_{I}\right|^{2}}{|I|}\left(1+\frac{\left|x-x_{I}\right|}{\ell(I)}\right)^{-(2 M-(d+\varepsilon))}\right)^{1 / 2} .
\end{gathered}
$$

\section{A few lemmas.}

Lemma 1. $|f(x)| \leq F^{*}(x)$.

Proof. Trivial.

Lemma 2. $G^{*}(x) \leq c g^{*}(x)$.

Proof. If $x \in I$ and $I^{\prime} \in S(I)$, then $\left|x-x_{I^{\prime}}\right| \leq\left|x-x_{I}\right|+\left|x_{I}-x_{I^{\prime}}\right| \leq$ $c\left|x_{I}-x_{I^{\prime}}\right|$, since $\left|x_{I}-x_{I^{\prime}}\right| \geq c \ell(I)$. Thus, for every $I^{\prime} \in S(I), 1+\mid x-$ $x_{I^{\prime}} \mid / \ell\left(I^{\prime}\right) \leq C\left(1+\left|x_{I^{\prime}}-x_{I}\right| / \ell\left(I^{\prime}\right)\right)$. This implies that $G(I) \leq c g^{*}(x)$.

Lemma 3. Let $0<\eta<0.1$. There is a $C=C(\eta, M, d)$ such that if $x \in I$ and $d(x, \partial I)>\eta \ell(I)$ then $C^{-1} G(I) \leq G(I, x) \leq C G(I)$.

Proof. Just note that if $x$ is as described and $I^{\prime} \in S(I)$ then $\mid x-$ $x_{I^{\prime}}|/| x_{I}-x_{I^{\prime}} \mid$ is bounded between two positive constants.

Lemma 4. Let $0<\eta<0.1$. There is a $C=C(\eta, M, d, \varepsilon)$ such that if $x \in I$ and $d(x, \partial I)>\eta \ell(I)$ then $|F(I)-F(I, x)| \leq C G(I)$.

Proof. Write

$$
\begin{aligned}
|F(I)-F(I, x)| \leq & \sum_{\substack{I^{\prime} \in S(I) \\
\ell\left(I^{\prime}\right) \geq \ell(I)}}\left|\lambda_{I^{\prime}}\right|\left|\phi_{\left(I^{\prime}\right)}(x)-\phi_{\left(I^{\prime}\right)}\left(x_{I}\right)\right| \\
& +\sum_{\substack{I^{\prime} \in S(I) \\
\ell\left(I^{\prime}\right)<\ell(I)}}\left|\lambda_{I^{\prime}}\right|\left(\left|\phi_{\left(I^{\prime}\right)}(x)\right|+\left|\phi_{\left(I^{\prime}\right)}\left(x_{I}\right)\right|\right) \\
= & (\mathrm{i})+(\mathrm{ii}) .
\end{aligned}
$$


Note that (i) has a minus sign where (ii) has a plus sign. We will use smoothness to bound (i), but only a size estimate to bound (ii). It is chiefly because of this latter fact that we are able to get away with so little smoothness in the family $\left\{\phi_{(I)}\right\}_{I}$.

We deal with (i) first. Let $I^{\prime} \in S(I)$ and suppose that $\ell\left(I^{\prime}\right) \geq \ell(I)$. By the smoothness condition on $\phi_{\left(I^{\prime}\right)}$,

$$
\left|\phi_{\left(I^{\prime}\right)}(x)-\phi_{\left(I^{\prime}\right)}\left(x_{I}\right)\right| \leq C_{d}\left(\frac{\ell(I)}{\ell\left(I^{\prime}\right)}\right)\left|I^{\prime}\right|^{-1 / 2}\left(1+\frac{\left|x_{I}-x_{I^{\prime}}\right|}{\ell\left(I^{\prime}\right)}\right)^{-M},
$$

for every such $I^{\prime}$. Therefore,

$$
\text { (i) } \leq C_{d} \sum_{\substack{I^{\prime} \in S(I) \\ \ell\left(I^{\prime}\right) \geq \ell(I)}}\left|\lambda_{I^{\prime}}\right|\left(\frac{\ell(I)}{\ell\left(I^{\prime}\right)}\right)\left|I^{\prime}\right|^{-1 / 2}\left(1+\frac{\left|x_{I}-x_{I^{\prime}}\right|}{\ell\left(I^{\prime}\right)}\right)^{-M} .
$$

Applying Cauchy-Schwarz, we get

$$
\begin{aligned}
(\mathrm{i}) & \leq c G(I)\left(\sum_{\substack{I^{\prime} \in S(I) \\
\ell\left(I^{\prime}\right) \geq \ell(I)}}\left(1+\frac{\left|x_{I}-x_{I^{\prime}}\right|}{\ell\left(I^{\prime}\right)}\right)^{-(d+\varepsilon)}\left(\frac{\ell(I)}{\ell\left(I^{\prime}\right)}\right)^{2}\right)^{1 / 2} \\
& \leq c G(I)\left(\sum_{k=0}^{\infty} 2^{-2 k} \sum_{\ell\left(I^{\prime}\right)=2^{k} \ell(I)}\left(1+\frac{\left|x_{I}-x_{I^{\prime}}\right|}{\ell\left(I^{\prime}\right)}\right)^{-(d+\varepsilon)}\right)^{1 / 2} .
\end{aligned}
$$

For each $k$, the sum

$$
\sum_{\ell\left(I^{\prime}\right)=2^{k} \ell(I)}\left(1+\frac{\left|x_{I}-x_{I^{\prime}}\right|}{\ell\left(I^{\prime}\right)}\right)^{-(d+\varepsilon)}
$$

is bounded by $C$, because, for each $n \geq 1$, there are at most $c 2^{\text {nd }}$ cubes $I^{\prime}$ in the sum such that $2^{n-1} \leq 1+\left|x_{I}-x_{I^{\prime}}\right| / \ell\left(I^{\prime}\right)<2^{n}$. Their contribution to the sum is no greater than $c 2^{-n \varepsilon}$.

Summing over $k$ now, we get that (i) $\leq c G(I)$.

To bound (ii), write $\mathbb{R}^{d}=I \cup\left(\cup_{j} I_{j}\right)$, where each $I_{j}$ is congruent to $I$ and is in $S(I)$. Here is where we use the hypothesis that $x \in I$ stays away from $\partial I$. Note that, if $I^{\prime} \subset I_{j}$, then $\left|x-x_{I^{\prime}}\right|,\left|x_{I}-x_{I^{\prime}}\right|$, and $\left|x_{I}-x_{I_{j}}\right|$ are comparable: i.e., the ratios

$$
\frac{\left|x-x_{I^{\prime}}\right|}{\left|x_{I}-x_{I_{j}}\right|}
$$


and

$$
\frac{\left|x-x_{I^{\prime}}\right|}{\left|x_{I}-x_{I^{\prime}}\right|}
$$

are both bounded above and below by positive constants that depend only on $d$ and $\eta$. Therefore, proceeding much as we did with (i)

$$
\begin{aligned}
\text { (ii) } & \leq c G(I)\left(\sum_{\substack{I^{\prime} \in S(I) \\
\ell\left(I^{\prime}\right)<\ell(I)}}\left(1+\frac{\left|x_{I}-x_{I^{\prime}}\right|}{\ell\left(I^{\prime}\right)}\right)^{-(d+\varepsilon)}\right)^{1 / 2} \\
& \leq c G(I)\left(\sum_{j} \sum_{I^{\prime} \subset I_{j}}\left(1+\frac{\left|x_{I}-x_{I^{\prime}}\right|}{\ell\left(I^{\prime}\right)}\right)^{-(d+\varepsilon)}\right)^{1 / 2} \\
& =c G(I)\left(\sum_{j} H(j)\right)^{1 / 2},
\end{aligned}
$$

where

$$
H(j)=\sum_{I^{\prime} \subset I_{j}}\left(1+\frac{\left|x_{I}-x_{I^{\prime}}\right|}{\ell\left(I^{\prime}\right)}\right)^{-(d+\varepsilon)} .
$$

Since, for each $j$,

$$
\left|x_{I}-x_{I_{j}}\right| \leq c_{d}\left|x_{I}-x_{I^{\prime}}\right|
$$

for all $I^{\prime} \in I_{j}$, we get

$$
\left(\frac{\left|x_{I}-x_{I_{j}}\right|}{\ell(I)}\right)\left(\frac{\ell(I)}{\ell\left(I^{\prime}\right)}\right) \leq c_{d} \frac{\left|x_{I}-x_{I^{\prime}}\right|}{\ell\left(I^{\prime}\right)}
$$

But, clearly,

$$
c\left(1+\frac{\left|x_{I}-x_{I_{j}}\right|}{\ell(I)}\right) \leq\left(\frac{\left|x_{I}-x_{I_{j}}\right|}{\ell(I)}\right)
$$

(because $\left.\left|x_{I}-x_{I_{j}}\right| \geq \ell(I)\right)$ and

$$
\frac{\left|x_{I}-x_{I^{\prime}}\right|}{\ell\left(I^{\prime}\right)} \leq\left(1+\frac{\left|x_{I}-x_{I^{\prime}}\right|}{\ell\left(I^{\prime}\right)}\right)
$$

Therefore

$$
\left(1+\frac{\left|x_{I}-x_{I^{\prime}}\right|}{\ell\left(I^{\prime}\right)}\right)^{-(d+\varepsilon)} \leq C\left(1+\frac{\left|x_{I}-x_{I_{j}}\right|}{\ell(I)}\right)^{-(d+\varepsilon)}\left(\frac{\ell\left(I^{\prime}\right)}{\ell(I)}\right)^{d+\varepsilon}
$$


for each $I^{\prime} \subset I_{j}$. Plugging this back into the sum for $H(j)$

$$
\begin{aligned}
H(j) & \leq c \sum_{I^{\prime} \subset I_{j}}\left(1+\frac{\left|x_{I}-x_{I_{j}}\right|}{\ell(I)}\right)^{-(d+\varepsilon)}\left(\frac{\ell\left(I^{\prime}\right)}{\ell(I)}\right)^{d+\varepsilon} \\
& =c\left(1+\frac{\left|x_{I}-x_{I_{j}}\right|}{\ell(I)}\right)^{-(d+\varepsilon)} \sum_{I^{\prime} \subset I_{j}}\left(\frac{\ell\left(I^{\prime}\right)}{\ell(I)}\right)^{d+\varepsilon} \\
& \leq c\left(1+\frac{\left|x_{I}-x_{I_{j}}\right|}{\ell(I)}\right)^{-(d+\varepsilon)} .
\end{aligned}
$$

There are no more than $c_{d} 2^{k d}$ cubes $I_{j}$ such that $2^{(k-1)}<\mid x_{I}-$ $x_{I_{j}} \mid / \ell(I) \leq 2^{k}$. The sum of the corresponding $H(j)$ 's is at most $c 2^{-k \varepsilon}$. Thus $\sum_{j} H(j) \leq C$, and the lemma is proved.

REMARK. In proving our main lemma, $\eta$ will be chosen so that $\{x \in$ $I: d(x, \partial I) \leq \eta \ell(I)\}$ has negligible measure.

Lemma 5. If $I^{*} \in N(I)$ then $G(I) \leq C(M, d) G\left(I^{*}\right)$.

Proof. By Lemma $3, G(I)=G\left(I, x_{I}\right) \leq C G\left(I, x_{I^{*}}\right)$. But $G\left(I, x_{I^{*}}\right) \leq$ $G\left(I^{*}\right)$.

Lemma 6. There is a positive constant $C$ such that the following holds: If $I^{*} \in N(I)$ then, for all $x \in I, G^{*}(x) \geq C G\left(I^{*}\right)$.

Proof. Let $L \subset I \backslash I^{*}$ be a cube. Let $J \in S\left(I^{*}\right)$. By the triangle inequality, $\left|x_{L}-x_{J}\right| \leq\left|x_{L}-x_{I^{*}}\right|+\left|x_{I^{*}}-x_{J}\right|$. But $\left|x_{L}-x_{I^{*}}\right| \leq c \ell(I) \leq$ $c\left|x_{I^{*}}-x_{J}\right|$. Therefore $\left|x_{L}-x_{J}\right| \leq C\left|x_{I^{*}}-x_{J}\right|$. Then,

$$
G\left(I^{*}\right)^{2} \leq C G(L)^{2}+\sum_{I^{\prime}: I^{\prime} \subset L} \frac{\left|\lambda_{I^{\prime}}\right|^{2}}{\left|I^{\prime}\right|}\left(1+\frac{\left|x_{I^{\prime}}-x_{I^{*}}\right|}{\ell\left(I^{\prime}\right)}\right)^{-(2 M-(d+\varepsilon))}
$$

for a fixed constant $C$. But the sum goes to zero as $|L|$ does.

Lemma 7. There is a $C=C(M, d, \varepsilon)$ such that if $I^{*} \in N(I)$ then $\left|F(I)-F\left(I^{*}\right)\right| \leq C G\left(I^{*}\right)$. 
ProOF. Write

$$
\begin{aligned}
\left|F(I)-F\left(I^{*}\right)\right| & \leq\left|F\left(I, x_{I}\right)-F\left(I, x_{I^{*}}\right)\right|+\left|F\left(I, x_{I^{*}}\right)-F\left(I^{*}, x_{I^{*}}\right)\right| \\
& \leq C G(I)+\left|F\left(I, x_{I^{*}}\right)-F\left(I^{*}, x_{I^{*}}\right)\right| \\
& \leq C G\left(I^{*}\right)+\left|F\left(I, x_{I^{*}}\right)-F\left(I^{*}, x_{I^{*}}\right)\right|
\end{aligned}
$$

where the second inequality follows from Lemma 4 and the third is from Lemma 5. The last term in the third inequality is less than or equal to

$$
\sum_{I^{\prime} \in S\left(I^{*}\right) \backslash S(I)}\left|\lambda_{I^{\prime}}\right|\left|I^{\prime}\right|^{-1 / 2}\left(1+\frac{\left|x_{I^{*}}-x_{I^{\prime}}\right|}{\ell\left(I^{\prime}\right)}\right)^{-M}
$$

which, by Cauchy-Schwarz, is less than or equal to

$$
G\left(I^{*}\right)\left(\sum_{\substack{I^{\prime}: I^{\prime} \subset I \\ I^{\prime} \not I^{*}}}\left(1+\frac{\left|x_{I^{*}}-x_{I^{\prime}}\right|}{\ell\left(I^{\prime}\right)}\right)^{-(d+\varepsilon)}\right)^{1 / 2} .
$$

A virtual repetition of the argument used to bound $H(j)$ in the proof of Lemma 4 shows that the sum in the brackets is bounded by a constant times

$$
\left(1+\frac{\left|x_{I^{*}}-x_{I}\right|}{\ell(I)}\right)^{-(d+\varepsilon)} \sum_{\substack{I^{\prime} ; I^{\prime} \subset I \\ I^{\prime} \not I^{*}}}\left(\frac{\ell\left(I^{\prime}\right)}{\ell(I)}\right)^{d+\varepsilon}
$$

which is less than or equal to $C$.

\section{The Main Lemma and its corollaries.}

Main Lemma. Let $I_{(0)}$ be a fixed dyadic cube and let $f=\sum_{I} \lambda_{I} \phi_{(I)}(x)$ be a finite linear sum from $\left\{\phi_{(I)}\right\}_{I}$. We assume that, for all I, either $\lambda_{I}=0$ or $I \subset I_{(0)}$. (Thus, we are only considering I's contained in $\left.I_{(0)}.\right)$ For every $\beta>0$ there is a $\gamma=\gamma(M, d, \beta, \varepsilon)>0$ such that

$$
\left|\left\{x \in I_{(0)}: F^{*}(x)>1, G^{*}(x) \leq \gamma\right\}\right| \leq \beta\left|I_{(0)}\right| .
$$

Proof of Main Lemma. This will be rather long.

Let $A$ be a large number, to be chosen shortly. Let $\left\{I_{j}\right\}_{j}$ be the maximal dyadic subcubes of $I_{(0)}$ such that there is an $I \in N\left(I_{j}\right)$ for 
which $G(I)>A \gamma$. By Lemma 6 , we have $G^{*}(x)>C A \gamma$ for all $x \in I_{j}$. Choose $A$ so that $C A>1$. Notice that, by maximality, we must have $G\left(I_{j}\right) \leq A \gamma$. Notice also that, if $x \notin \cup_{j} I_{j}$, then $G^{*}(x) \leq A \gamma$. These two facts imply that, for any $x \in I_{(0)}$,

$$
\sum_{\substack{I: x \in I \\ \text { for all } j\left(I \not \subset I_{j}\right)}} \frac{\left|\lambda_{I}\right|^{2}}{|I|} \leq C(M, d)(A \gamma)^{2} .
$$

Now observe that if $x \in\left\{x \in I_{(0)}: F^{*}(x)>1, G^{*}(x) \leq \gamma\right\}$ then $x$ must belong to some cube $I$ such that $|F(I)|>1$ and $I$ is not a subset of any $I_{j}$ (because $G^{*}(x)>\gamma$ on $\left.\cup_{j} I_{j}\right)$. Let $\left\{J_{k}\right\}_{k}$ be the maximal cubes with both these properties. Set $\left\{Q_{j}^{*}\right\}=\left\{I_{j}\right\}_{j} \cup\left\{J_{k}\right\}_{k}$, the union of these two sets of cubes (so, $\left\{Q_{j}^{*}\right\}$ is a collection of cubes), and let $\left\{Q_{j}\right\}_{j} \subset\left\{Q_{j}^{*}\right\}$ be the corresponding subfamily of maximal cubes.

We observe that if $x \in\left\{x \in I_{(0)}: F^{*}(x)>1, G^{*}(x) \leq \gamma\right\}$ then $x$ must belong to some cube $Q_{j}$ such that $\left|F\left(Q_{j}\right)\right|>1$. We also note that $G\left(Q_{j}\right) \leq A \gamma$ for all $j$ and that, if $x \notin \cup_{j} Q_{j}$, then $G^{*}(x) \leq A \gamma$.

So far, (almost) everything we have done has consisted of fairly standard (if somewhat technical) estimates. We now come to the SECOND MAIN IDEA; i.e., the splitting of the sum.

Let $\mathcal{F}_{1}=\left\{I\right.$ : for all $\left.j\left(I \not \subset Q_{j}\right)\right\}$ and $\mathcal{F}_{2}=\left\{I: I \subset Q_{j}\right.$ for some $\left.j\right\}$. Every $I \subset I_{(0)}$ belongs to either $\mathcal{F}_{1}$ or $\mathcal{F}_{2}$. Write $f=f_{1}+f_{2}$, where $f_{l}=\sum_{I: I \in \mathcal{F}_{l}} \lambda_{I} \phi_{(I)}(x)$. We similarly define $F_{l}(I, x), F_{l}(I), G_{l}^{*}(x)$, etc., e.g.

$$
\begin{aligned}
& F_{1}(I, x)=\sum_{\substack{I^{\prime} \in S(I) \\
I^{\prime} \in \mathcal{F}_{1}}} \lambda_{I^{\prime}} \phi_{\left(I^{\prime}\right)}(x), \\
& F_{2}(I, x)=\sum_{\substack{I^{\prime} \in S(I) \\
I^{\prime} \in \mathcal{F}_{2}}} \lambda_{I^{\prime}} \phi_{\left(I^{\prime}\right)}(x),
\end{aligned}
$$

both defined only for $x \in I$. Notice that $G_{l}(I) \leq G(I)$.

Now, it is clear that

$$
\begin{aligned}
\mid\left\{x \in I_{(0)}: F^{*}\right. & \left.(x)>1, G^{*}(x) \leq \gamma\right\} \mid \\
& \leq \sum_{j^{\prime}:\left|F\left(Q_{j^{\prime}}\right)\right|>1}\left|Q_{j^{\prime}}\right| \\
& \leq \sum_{j^{\prime}:\left|F_{1}\left(Q_{j^{\prime}}\right)\right|>1 / 2}\left|Q_{j^{\prime}}\right|+\sum_{j^{\prime}:\left|F_{2}\left(Q_{j^{\prime}}\right)\right|>1 / 2}\left|Q_{j^{\prime}}\right| \\
& =(\mathrm{i})+(\mathrm{ii}) .
\end{aligned}
$$


We need to estimate (i) and (ii). Define, for $Q$ a cube, $C(Q)=\{x: \mid x-$ $\left.x_{Q} \mid \leq 0.1 \ell(Q)\right\}$, the "center portion" of $Q$. Notice that $|Q| \leq c_{d}|C(Q)|$. Thus

$$
\text { (i) } \leq c_{d} \sum_{j^{\prime}:\left|F_{1}\left(Q_{j^{\prime}}\right)\right|>1 / 2}\left|C\left(Q_{j^{\prime}}\right)\right|,
$$

and an analogous estimate holds for (ii).

Let $Q_{j^{\prime}}$ be a cube occurring in the sum for (i). By Lemma 4, if $x \in C\left(Q_{j^{\prime}}\right),\left|F_{1}\left(Q_{j^{\prime}}\right)-F_{1}\left(Q_{j^{\prime}}, x\right)\right| \leq C(M, d, \varepsilon) G\left(Q_{j^{\prime}}\right)$. But $G\left(Q_{j^{\prime}}\right) \leq$ $A \gamma$ and $\left|F_{1}\left(Q_{j^{\prime}}\right)\right|>1 / 2$. If $\gamma$ is taken small enough, these force $\left|F_{1}\left(Q_{j^{\prime}}, x\right)\right|>0.25$ for all $x \in C\left(Q_{j^{\prime}}\right)$. Therefore,

$$
\text { (i) } \leq c_{d} \sum_{j}\left|\left\{x \in C\left(Q_{j}\right):\left|F_{1}\left(Q_{j}, x\right)\right|>0.25\right\}\right|,
$$

and a similar estimate holds for (ii).

Let us temporarily restrict our attention to (i). We now make a curious observation: If $x \in Q_{k}$ then $F_{1}\left(Q_{k}, x\right)=f_{1}(x)$. The proof comes from working it out

$$
\begin{aligned}
f_{1}(x) & =\sum_{I \in \mathcal{F}_{1}} \lambda_{I} \phi_{(I)}(x) \\
& =\sum_{I: \text { for all } j\left(I \not \subset Q_{j}\right)} \lambda_{I} \phi_{(I)}(x) \\
& =\sum_{\substack{I: I \in \mathcal{F}_{1} \\
I \in S\left(Q_{k}\right)}} \lambda_{I} \phi_{(I)}(x) \\
& =F_{1}\left(Q_{k}, x\right) .
\end{aligned}
$$

(It is helpful to recall the definition of $S\left(Q_{k}\right)=\left\{I: I \not \subset Q_{k}\right\}$.)

(This "curious observation" is (sort of) why the proof works: this is where the two main ideas link up.)

Therefore,

$$
\text { (i) } \leq c\left|\left\{\left|f_{1}(x)\right|>0.25\right\}\right| \text {. }
$$

However, by our hypothesis (0.2),

$$
\int\left|f_{1}\right|^{2} d x \leq C \sum_{I: I \in \mathcal{F}_{1}}\left|\lambda_{I}\right|^{2}=\int\left(\sum_{\substack{I: x \in I \\ I \in \mathcal{F}_{1}}} \frac{\left|\lambda_{I}\right|^{2}}{|I|}\right) d x .
$$


As observed above, the quantity

$$
\sum_{\substack{I: x \in I \\ I \in \mathcal{F}_{1}}} \frac{\left|\lambda_{I}\right|^{2}}{|I|}
$$

is less than or equal to $C(A \gamma)^{2}$ everwhere. A good bound on (i) now follows from Chebyshev's inequality.

Let's look at (ii) now. For each $j$, let $E_{j}=\left\{I: I \subset Q_{j}\right\}$. Notice that $\mathcal{F}_{2}=\cup_{j} E_{j}$. For $\tau>1$ let $\tau I$ denote $I$ 's $\tau$-fold dilate. If $\tau$ is taken close enough to 1 (depending only on $d$ ), $|\tau I \backslash I|<(\beta / 3)|I|$. Fix such a $\tau$ and set $D=\cup_{j}\left(\tau Q_{j} \backslash Q_{j}\right)$. Then $|D|<(\beta / 3)\left|I_{(0)}\right|$. We only need to show that

$$
c_{d} \sum_{j}\left|\left\{x \in C\left(Q_{j}\right) \backslash D:\left|F_{2}\left(Q_{j}, x\right)\right|>0.25\right\}\right| \leq \frac{\beta}{3}\left|I_{(0)}\right| .
$$

Fix a $j$ in the preceding sum and let $x \in C\left(Q_{j}\right) \backslash D$. The function $\left|F_{2}\left(Q_{j}, x\right)\right|$ is less than or equal to

$$
\sum_{k: k \neq j} \sum_{I: I \in E_{k}} \frac{\left|\lambda_{I}\right|}{\sqrt{|I|}}\left(1+\frac{\left|x-x_{I}\right|}{\ell(I)}\right)^{-M}
$$

which, by Cauchy-Schwarz, is bounded by

$$
\begin{gathered}
\left(\sum_{k: k \neq j} \sum_{I: I \in E_{k}} \frac{\left|\lambda_{I}\right|^{2}}{|I|}\left(1+\frac{\left|x-x_{I}\right|}{\ell(I)}\right)^{-(2 M-(d+\varepsilon))}\right)^{1 / 2} \\
\cdot\left(\sum_{k: k \neq j} \sum_{I: I \in E_{k}}\left(1+\frac{\left|x-x_{I}\right|}{\ell(I)}\right)^{-(d+\varepsilon)}\right)^{1 / 2} .
\end{gathered}
$$

The first factor in the preceding product is less than or equal to $G\left(Q_{j}, x\right)$ $\leq C G\left(Q_{j}\right) \leq c A \gamma$. Let us now look at the second factor.

Consider

$$
\sum_{k: k \neq j} \sum_{I: I \in E_{k}}\left(1+\frac{\left|x-x_{I}\right|}{\ell(I)}\right)^{-(d+\varepsilon)} .
$$

Fix a $k \neq j$ in the sum. Since $Q_{k} \cap Q_{j}=\varnothing$ and $x \notin D$, we must have $\left|x-x_{I}\right| \geq c\left|x-x_{Q_{k}}\right|$ for every $I \in E_{k}$, where the constant $c$ only 
depends on $\tau$ and $d$. Therefore

$$
\begin{aligned}
1+\frac{\left|x-x_{I}\right|}{\ell(I)} & \geq \frac{\left|x-x_{I}\right|}{\ell(I)} \\
& \geq c \frac{\left|x-x_{Q_{k}}\right|}{\ell\left(Q_{k}\right)}\left(\frac{\ell\left(Q_{k}\right)}{\ell(I)}\right) \\
& \geq c\left(1+\frac{\left|x-x_{Q_{k}}\right|}{\ell\left(Q_{k}\right)}\right)\left(\frac{\ell\left(Q_{k}\right)}{\ell(I)}\right),
\end{aligned}
$$

where the last inequality follows because $\left|x-x_{Q_{k}}\right| \geq 0.5 \ell\left(Q_{k}\right)$ (recall that $x \notin Q_{k}$ ). Thus

$$
\left(1+\frac{\left|x-x_{I}\right|}{\ell(I)}\right)^{-(d+\varepsilon)} \leq C\left(1+\frac{\left|x-x_{Q_{k}}\right|}{\ell\left(Q_{k}\right)}\right)^{-(d+\varepsilon)}\left(\frac{\ell(I)}{\ell\left(Q_{k}\right)}\right)^{d+\varepsilon} .
$$

Therefore, if $x \in C\left(Q_{j}\right) \backslash D$,

$$
\begin{aligned}
\left|F_{2}\left(Q_{j}, x\right)\right| & \leq \operatorname{cA\gamma }\left(\sum_{k: k \neq j}\left(1+\frac{\left|x-x_{Q_{k}}\right|}{\ell\left(Q_{k}\right)}\right)^{-(d+\varepsilon)} \sum_{I \in E_{k}}\left(\frac{\ell(I)}{\ell\left(Q_{k}\right)}\right)^{d+\varepsilon}\right)^{1 / 2} \\
& \leq C A \gamma\left(\sum_{k}\left(1+\frac{\left|x-x_{Q_{k}}\right|}{\ell\left(Q_{k}\right)}\right)^{-(d+\varepsilon)}\right)^{1 / 2} .
\end{aligned}
$$

This implies that

$$
\begin{aligned}
\sum_{j} \int_{C\left(Q_{j}\right) \backslash D}\left|F\left(Q_{j}, x\right)\right|^{2} d x & \leq(C A \gamma)^{2} \int_{I_{(0)}} \sum_{k}\left(1+\frac{\left|x-x_{Q_{k}}\right|}{\ell\left(Q_{k}\right)}\right)^{-(d+\varepsilon)} \\
& \leq(C A \gamma)^{2} \sum_{k}\left|Q_{k}\right| \\
& \leq(C A \gamma)^{2}\left|I_{(0)}\right|
\end{aligned}
$$

Now the bound on (ii) follows (for $\gamma$ sufficiently small) by Chebyshev's inequality. The Main Lemma is proved.

Corollary of the Main Lemma. Let $\sigma$ be an $A_{\infty}$ weight. For every $\beta>0$ there is $a \gamma>0$ such that for all $\lambda>0$ and any finite sum $f=\sum_{I} \lambda_{I} \phi_{(I)}(x)$ (as described in Section 1$)$,

$$
\sigma\left(\left\{x: F^{*}(x)>2 \lambda, G^{*}(x) \leq \gamma \lambda\right\}\right) \leq \beta \sigma\left(\left\{x: F^{*}(x)>\lambda\right\}\right) .
$$


Proof of THE COROLlaRY. Let $\left\{I_{j}\right\}_{j}$ be the maximal dyadic cubes such that $\left|F\left(I_{j}\right)\right|>\lambda$. Then $\left\{x: F^{*}(x)>\lambda\right\}=\cup_{j} I_{j}$. It will be enough to show that

$$
\sigma\left(\left\{x \in I_{j}: F^{*}(x)>2 \lambda, G^{*}(x) \leq \gamma \lambda\right\}\right) \leq \beta \sigma\left(I_{j}\right),
$$

for each $j$, where $\gamma$ does not depend on $j$. Because $\sigma \in A_{\infty}$, it will be enough to show

$$
\left|\left\{x \in I_{j}: F^{*}(x)>2 \lambda, G^{*}(x) \leq \gamma \lambda\right\}\right| \leq \beta\left|I_{j}\right|,
$$

for some smaller (but fixed) value of $\beta$.

Let $\eta>0$ be so small that

$$
\left|\left\{x \in I_{j}: d\left(x, \partial I_{j}\right) \leq \eta \ell\left(I_{j}\right)\right\}\right| \leq \frac{\beta}{3}\left|I_{j}\right|
$$

Let $\tilde{I}_{j}$ be $I_{j}$ 's dyadic double (i.e., $\left.I_{j} \in N\left(\tilde{I}_{j}\right)\right)$. We have that $\left|F\left(\tilde{I}_{j}\right)\right| \leq$ $\lambda$. By Lemma $7,\left|F\left(I_{j}\right)-F\left(\tilde{I}_{j}\right)\right| \leq C G\left(I_{j}\right)$. By Lemma 4 , we also have that $\left|F\left(I_{j}\right)-F\left(I_{j}, x\right)\right| \leq C_{\eta} G\left(I_{j}\right)$ for all $x \in I_{j}$ such that $d\left(x, \partial I_{j}\right)>$ $\eta \ell\left(I_{j}\right)$. Thus, by taking $\gamma$ small enough, we can assume that either the left-hand side of (3.1) is zero or else $\left|F\left(I_{j}, x\right)\right| \leq 1.1 \lambda$ for all $x$ such that $d\left(x, \partial I_{j}\right)>\eta \ell\left(I_{j}\right)$. We henceforth assume we are in the second case. Write $h=\sum_{I \subset I_{j}} \lambda_{I} \phi_{(I)}$. We are going to apply the Main Theorem to $h$ on the cube $I_{j}$. Set

$$
\begin{gathered}
H(I, x)=\sum_{\substack{I^{\prime}: I^{\prime} \in S(I) \\
I^{\prime} \subset I_{j}}} \lambda_{I^{\prime}} \phi_{\left(I^{\prime}\right)}(x), \\
H(I)=H\left(I, x_{I}\right), \\
H^{*}(x)=\sup _{I \ni x}|H(I)| .
\end{gathered}
$$

It is clear that

$$
\begin{aligned}
\left\{x \in I_{j}: F^{*}(x)>2 \lambda, G^{*}(x)\right. & \leq \gamma \lambda\} \\
\subset & \left\{x \in I_{j}: H^{*}(x)>0.9 \lambda, G^{*}(x) \leq \gamma \lambda\right\} \\
& \cup\left\{x \in I_{j}: d\left(x, \partial I_{j}\right) \leq \eta \ell\left(I_{j}\right)\right\} .
\end{aligned}
$$


But the second set on the right has measure at most $(\beta / 3)\left|I_{j}\right|$. By rescaling (dividing by $0.9 \lambda$ ) and applying the Main Lemma, so does the first set on the right. This proves the application.

We are now able to prove:

Theorem A. Let $0<p<\infty$ and let $\sigma \in A_{\infty}$. For every finite sum

$$
f=\sum_{I} \lambda_{I} \phi_{(I)}(x)
$$

as described in the introduction,

$$
\int_{\mathbb{R}^{d}}|f|^{p} \sigma d x \leq C \int_{\mathbb{R}^{d}}\left(g^{*}(x)\right)^{p} \sigma d x .
$$

In particular,

$$
\int_{\mathbb{R}^{d}}|f|^{2} \sigma d x \leq C \sum_{I} \frac{\left|\lambda_{I}\right|^{2}}{|I|} \int_{\mathbb{R}^{d}} \frac{\sigma(x)}{\left(1+\frac{\left|x-x_{I}\right|}{\ell(I)}\right)^{2 M-(d+\varepsilon)}} d x .
$$

Proof of Theorem A. If $x$ is large and $x \in I$ then $\left|x_{I}\right| \geq c|x|$. Thus,

$$
F^{*}(x) \leq c|x|^{-M} \leq c G^{*}(x) \leq c g^{*}(x)
$$

for large $x$; so, without loss of generality, we may assume that $F^{*} \in$ $L^{p}(\sigma)$. Now the Corollary implies that

$$
\int_{\mathbb{R}}\left(F^{*}(x)\right)^{p} \sigma d x \leq C \int_{\mathbb{R}^{d}}\left(G^{*}(x)\right)^{p} \sigma d x .
$$

Lemmas 1 and 2 finish the proof.

Corollary of Theorem A. Let $p, \sigma$, and $\left\{\phi_{(I)}\right\}_{I}$ be as in Theorem A, and let $\left\{\lambda_{I}\right\}_{I \in \mathcal{D}} \subset \mathbb{R}$. If $\left\{\mathcal{D}_{n}\right\}$ is a nested, increasing sequence of finite subsets of $\mathcal{D}$ such that

$$
f(x) \equiv \lim _{n \rightarrow \infty} \sum_{I \in \mathcal{D}_{n}} \lambda_{I} \phi_{(I)}(x)
$$

exists almost everwhere, then

$$
\int_{\mathbb{R}^{d}}|f|^{p} \sigma d x \leq C \int_{\mathbb{R}^{d}}\left(g^{*}(x)\right)^{p} \sigma d x
$$


where the constant $C$ is the same as in Theorem $A$.

Proof. Fatou's Lemma.

The reader should notice that, although we only require $M>d / 2$, we will almost always want $2 M-(d+\varepsilon)>d$, which requires $M>d$. In order to get away with nothing stronger than $M>d / 2$ (in practice, as it were), we would have to raise the exponent of decay in the definition of $g^{*}(x)$ to $2 M-\varepsilon$. Alas, this is impossible, at least in the generality of Theorem A. The reader is referred to [W2] for the counterexample.

\section{Appendix.}

We remind the reader that this proof's real payoff comes in the decomposition, and that almost-orthogonality can be obtained under slightly weaker hypotheses [FJW].

Almost-orthogonality Lemma. If the family $\left\{\phi_{(I)}\right\}_{I}$ satisfies (0.1), (0.3), and (0.4), then it satisfies (0.2), modulo a multiplicative constant, depending only on $M$ and $d$.

Proof. See [U, Lemmas 3.5 and 3.3] (in that order). By (the proof of) Lemma 3.5 , each of our $\phi_{(I)}$ 's can be decomposed

$$
\phi_{(I)}=C(M, d) \sum_{j=0}^{\infty} 2^{-M j} \phi_{(I), j},
$$

where each $\phi_{(I), j}$ is smooth and satisfies

$$
\begin{gathered}
\operatorname{supp} \phi_{(I), j} \subset 2^{j} I, \\
\left\|\phi_{(I), j}\right\|_{\infty} \leq|I|^{-1 / 2}, \\
\left\|\nabla \phi_{(I), j}\right\|_{\infty} \leq\left(2^{j} \ell(I)\right)^{-1}|I|^{-1 / 2}, \\
\int \phi_{(I), j} d x=0
\end{gathered}
$$

$\left(2^{j} I\right.$ is the $2^{j}$-fold dilate of $\left.I\right)$. 
Uchiyama proves his Lemma 3.5 for $M=d+1$, but it is easy to see that the proof goes through for any $M>d$. The reader should notice that our $\phi_{(I)}$ 's differ from Uchiyama's by factors of $|I|^{1 / 2}$.

By Lemma 3.3, we have, for each $j$,

$$
\left\|\sum_{I} \lambda_{I} \phi_{(I), j}\right\|_{2} \leq C_{d} 2^{j d}\left(\sum_{I}\left|\lambda_{I}\right|^{2}\right)^{1 / 2} .
$$

Therefore, if

$$
h=\sum_{I} \lambda_{I} \phi_{(I)}
$$

is a finite linear sum from $\left\{\phi_{(I)}\right\}_{I}$,

$$
\begin{aligned}
\|h\|_{2} & \leq C \sum_{j} 2^{-M j}\left\|\sum_{I} \lambda_{I} \phi_{(I), j}\right\|_{2} \\
& \leq C\left(\sum_{I}\left|\lambda_{I}\right|^{2}\right)^{1 / 2} \sum_{j} 2^{-M j} 2^{j d} \\
& \leq C\left(\sum_{I}\left|\lambda_{I}\right|^{2}\right)^{1 / 2},
\end{aligned}
$$

since $M>d$.

Acknowledgements. We wish to express our deep gratitude to Richard Wheeden, for allowing the author to talk through some early (and unsuccessful) versions of this proof on his blackboard at Rutgers, when the two of us were supposed to be working on something else.

\section{References.}

[FJW] Frazier, M., Jawerth, B., Weiss, G., Littlewood-Paley theory and the study of function spaces. American Mathematical Society, 1991.

[St1] Stein, E. M., Singular Integrals and Differentiability Properties of Functions. Princeton University Press, 1970.

[St2] Stein, E. M., Harmonic Analysis. Princeton University Press, 1993.

[W1] Wilson, J. M., A semi-discrete Littlewood-Paley inequality. Submitted for publication. 
[W2] Wilson, J. M., Note on a Littlewood-Paley inequality. To appear in Proc. Amer. Math. Soc.

[U] Uchiyama, A., A constructive proof of the Fefferman-Stein decomposition of $\operatorname{BMO}\left(\mathbb{R}^{n}\right)$. Acta Math. 148 (1982), 215-241.

Recibido: 19 de enero de 1.999

J. Michael Wilson*

Department of Mathematics

University of Vermont

Burlington, Vermont 05405, U.S.A.

wils on@math.uvm. edu

\footnotetext{
* Supported by NSF grant DMS 9501107.
} 